\title{
Adaptive Plasticity Under Adverse Listening Conditions is Disrupted in Developmental Dyslexia
}

\author{
Yafit Gabay ${ }^{1,2, *}$ (D) and Lori L. Holt ${ }^{3,4, *}$ \\ ${ }^{1}$ Department of Special Education, University of Haifa, Haifa, Israel \\ ${ }^{2}$ Edmond J. Safra Brain Research Center for the Study of Learning Disabilities, Haifa, Israel \\ ${ }^{3}$ Department of Psychology, Carnegie Mellon University, Pittsburgh, PA, USA \\ ${ }^{4}$ Neuroscience Institute, Carnegie Mellon University, Pittsburgh, PA, USA
}

(Received February 11, 2020; Final Revision April 16, 2020; Accepted May 19, 2020; First Published Online August 7, 2020)

\begin{abstract}
Objective: Acoustic distortions to the speech signal impair spoken language recognition, but healthy listeners exhibit adaptive plasticity consistent with rapid adjustments in how the distorted speech input maps to speech representations, perhaps through engagement of supervised error-driven learning. This puts adaptive plasticity in speech perception in an interesting position with regard to developmental dyslexia inasmuch as dyslexia impacts speech processing and may involve dysfunction in neurobiological systems hypothesized to be involved in adaptive plasticity. Method: Here, we examined typical young adult listeners $(N=17)$, and those with dyslexia $(N=16)$, as they reported the identity of native-language monosyllabic spoken words to which signal processing had been applied to create a systematic acoustic distortion. During training, all participants experienced incremental signal distortion increases to mildly distorted speech along with orthographic and auditory feedback indicating word identity following response across a brief, 250-trial training block. During pretest and posttest phases, no feedback was provided to participants. Results: Word recognition across severely distorted speech was poor at pretest and equivalent across groups. Training led to improved word recognition for the most severely distorted speech at posttest, with evidence that adaptive plasticity generalized to support recognition of new tokens not previously experienced under distortion. However, training-related recognition gains for listeners with dyslexia were significantly less robust than for control listeners. Conclusions: Less efficient adaptive plasticity to speech distortions may impact the ability of individuals with dyslexia to deal with variability arising from sources like acoustic noise and foreign-accented speech.
\end{abstract}

Keywords: Adaptive plasticity, Developmental dyslexia, Procedural learning, Speech recognition, Supervised learning

\section{INTRODUCTION}

Mature listeners are well attuned to the subtle relationships of acoustic dimensions that characterize native speech input. Yet, any given listening context can involve speech that deviates from these expected patterns. A talker may speak with an unusual accent or dialect, or environmental factors may create acoustic distortion. These adverse listening conditions relate to any situation in which the acoustic signal is distorted, degraded, or distinct from its typical form. This can arise from intrinsic (accented or dysfluent speech) as well as extrinsic (background noise or distortion over a channel) properties of speech. Such conditions can complicate the mapping of the acoustic speech signal onto meaningful native-language

*Correspondence and reprint requests to: Yafit Gabay, Ph.D., University of Haifa, Mount Carmel, Haifa 31905, Israel. E-mail: ygabay@edu.haifa.ac.il; Lori L. Holt, Ph.D., Carnegie Mellon University, Pittsburgh, PA, USA. E-mail: loriholt@cmu.edu sounds and words and negatively impact comprehension (Cooper, Brouwer, \& Bradlow, 2015; Kalikow, Stevens, \& Elliott, 1977). Even so, when listeners encounter speech under adverse listening conditions, adaptive perceptual adjustments can improve comprehension over time (Altmann \& Young, 1993; Francis, Baldwin, \& Nusbaum, 2000; Greenspan, Nusbaum, \& Pisoni, 1988; Liss, Spitzer, Caviness, \& Adler, 2002; Norris, McQueen, \& Cutler, 2003; Pallier, SebastianGallés, Dupoux, Christophe, \& Mehler, 1998; Schwab, Nusbaum, \& Pisoni, 1985). These improvements are thought of as adaptive plasticity in which exposure to distorted or degraded speech leads to perceptual adaptations that lead to more effectively subsequent mapping of the distorted speech. Adaptive plasticity has been observed across many different speech signal distortions, including foreign-accented, synthetic, time-compressed, noise-vocoded, and dysarthric speech (e.g., Altmann \& Young, 1993; Banai \& Lavner, 2012, 2014; Bradlow \& Bent, 2008; Cainer, James, \& Rajan, 2008; 
Clarke \& Garrett, 2004; Davis, Johnsrude, Hervais-Adelman, Taylor, \& McGettigan, 2005; Gabay, Karni, \& Banai, 2017; Liss et al., 2002; Schwab et al., 1985).

The mechanistic origins of adaptive plasticity are not yet well understood and there is debate about whether phenomena observed across different distortions and tasks arise from common mechanisms. However, the presence of information that disambiguates speech that deviates from the norm emerges as an important common factor. Speech recognition improves more when exposure to distorted speech is accompanied with disambiguating information like a written version of the speech (Francis, Nusbaum, \& Fenn, 2007; Loebach, Pisoni, \& Svirsky, 2010; Schwab et al., 1985), or a clear (undistorted) acoustic version of the speech (Davis et al., 2005; Hervais-Adelman, Davis, Johnsrude, \& Carlyon, 2008). Even so, adaptive plasticity is sometimes observed even when there are no external information sources available to disambiguate distorted speech (Altmann \& Young, 1993; Clarke \& Garrett, 2004; Liss et al., 2002; Pallier et al., 1998). Guediche, Fiez, and Holt (2016) make the case that both external (e.g., written feedback) and internal (e.g., word knowledge) information sources can be used to generate predictions about the correct mapping of distorted speech input. Their results demonstrate that mere exposure is sufficient to drive adaptive plasticity for less-severely distorted speech input and that external information sources enhance adaptive plasticity above and beyond this only when input signals are so severely degraded that they cannot provide access to internal predictions to drive adaptive plasticity in speech perception via internal information.

Several groups have argued that results like these are consistent with generation of an error signal when there is a discrepancy between the sensory input predicted by disambiguated signal and the actual (distorted) speech experienced (Guediche, Holt, Laurent, Lim, \& Fiez, 2015; Vroomen, Baart, Murray, \& Wallace, 2012; Vroomen, van Linden, De Gelder, \& Bertelson, 2007). This signal, in turn, may drive adaptive plasticity through supervised error-driven learning that impacts the mapping of acoustics to speech representations (see Guediche et al., 2015). Consistent with the possibility that error-driven learning may play a role in adaptive plasticity in speech perception, Guediche et al. (2015) present evidence that a neurobiological system implicated in supervised error-driven learning in other domains (Wolpert, Diedrichsen, \& Flanagan, 2011; Wolpert \& Kawato, 1998; Wolpert, Miall, \& Kawato, 1998), namely the cerebellum, is involved in adaptive plasticity in speech perception.

This situates adaptive plasticity in speech perception in an interesting position with regard to developmental dyslexia, one of the most common neurodevelopmental disorders (Peterson \& Pennington, 2015). Although the common view suggests that developmental dyslexia arises from a phonological impairment (Ramus \& Szenkovits, 2008; Snowling, 1998), this stance has been criticized for its inability to explain broader deficits not restricted to phonological information such as motor impairments (Orban, Lungu, \& Doyon, 2008; Stoodley, Harrison, \& Stein, 2006) visual and auditory deficits (Farmer \& Klein, 1995), and procedural learning impairments (Gabay, Schiff, \& Vakil, 2012a, 2012c; Gabay, Vakil, Schiff, \& Holt, 2015; Hedenius et al., 2013; Howard, Howard, Japikse, \& Eden, 2006; Lum, Ullman, \& Conti-Ramsden, 2013; Pavlidou \& Williams, 2014; Stoodley, Ray, Jack, \& Stein, 2008; Stoodley et al., 2006; Vicari et al., 2005). It is possible that phonological impairments are a symptom, rather than a cause, of developmental dyslexia.

A recent explanatory framework posits that developmental dyslexia may be related to impairments to the procedural learning system (Nicolson \& Fawcett, 2010; Nicolson \& Fawcett, 2011; Nicolson, Fawcett, \& Dean, 2001; Ullman, 2004; Ullman, Earle, Walenski, \& Janacsek, 2020) related to knowledge honed through automatization of various skills ("learning how") and contrasted with declarative learning ("learning that") which represents memory for events and facts about the world (Knowlton, Siegel, \& Moody, 2017). Procedural learning impairments in developmental dyslexia are hypothesized to affect the automatization of various skills and subskills ultimately required for successful reading.

A growing body of studies supports this possibility through demonstrations of procedural learning deficits in nonphonological and even non-language domains in developmental dyslexia. For example, impairments in motor sequence learning (Gabay et al., 2012a; Lum et al., 2013), nonspeech/visual statistical learning (Gabay, Thiessen, \& Holt, 2015; Singh, Walk, \& Conway, 2018), and probabilistic learning in a visual task (Gabay, Vakil, et al., 2015) have been observed in developmental dyslexia. Furthermore, core neurobiological components of the procedural learning system, such as the cerebellum (Fernandez et al., 2016; Nicolson et al., 1999; Pernet, Poline, Demonet, \& Rousselet, 2009; Rae et al., 1998; Stanberry et al., 2006; Stoodley, 2014), and the basal ganglia (Brunswick, McCrory, Price, Frith, \& Frith, 1999; Kita et al., 2013; Paulesu et al., 1996), have been found to be affected in dyslexia. In addition, people with developmental dyslexia exhibit impairments in tasks that tap cerebellar function, including eye blink conditioning (Nicolson, Daum, Schugens, Fawcett, \& Schulz, 2002) and motor adaptation (Brookes, Nicolson, \& Fawcett, 2007). However, most studies broadly examining procedural learning in developmental dyslexia, and cerebellar dysfunction specifically, have concentrated on motor learning (Brookes et al., 2007; Gabay et al., 2012a; Gabay, Schiff, \& Vakil, 2012b; Hedenius et al., 2013; Howard et al., 2006; Lum et al., 2013; Menghini, Hagberg, Caltagirone, Petrosini, \& Vicari, 2006; Needle, Nicolson, \& Fawcett, 2015; Stoodley et al., 2008; Stoodley et al., 2006; Vicari, Marotta, Menghini, Molinari, \& Petrosini, 2003; Yang \& Hong-Yan, 2011). Given increasing evidence that implicates the cerebellum in purely auditory tasks that do not involve motor processing (Petacchi, Laird, Fox, \& Bower, 2005), prediction error signals in speech perception (Rothermich \& Kotz, 2013) and the adaptive plasticity of speech perception phenomena described above (Guediche et al., 2015), there is the potential that disrupted cerebellar processing in developmental 
dyslexia (Alvarez \& Fiez, 2018; Fawcett \& Nicolson, 2008; Pernet et al., 2009) could impact perception, as well. On this hypothesis, we conducted a behavioral study of adaptive plasticity in speech perception following the methodological approach of Guediche and colleagues (Guediche, Blumstein, Fiez, \& Holt, 2014; Guediche et al., 2015, 2016) across adults with developmental dyslexia, and matched control participants. This approach has the advantage of introducing distortion to spectral acoustic information, leaving temporal information largely intact to protect against the possibility of the distortion manipulation interacting with reported temporal auditory processing impairments in dyslexia (Cohen-Mimran \& Sapir, 2007; Farmer \& Klein, 1995; Gabay, Najjar, \& Reinisch, 2019; Van Ingelghem et al., 2001).

\section{METHODS}

\section{Participants}

Thirty-four participants took part in the study $(N=17$ adults with developmental dyslexia; $N=17$ typical readers). All were university students in Pittsburgh, PA. The dyslexia group was recruited from the office of disability resources at Carnegie Mellon University. A documented diagnosis of a comorbid learning disability, such as attention deficit hyperactivity disorder or specific language impairment, or any sensory or neurological impairment was an exclusion criterion. All participants with dyslexia reported having substantial difficulties with reading and writing skills during school entry. The inclusion criteria for the dyslexia group were (a) a formal diagnosis of dyslexia by a qualified psychologist, as indicated by previous and recent test records of the participant and (b) a score of below the 50th percentile on at least two word-level-reading tests (untimed and timed tests of word reading and decoding skills, similar to the study of Thompson et al. [2018]). The control group consisted of individuals with no reading impairments (i.e., performance above the inclusion criteria of the dyslexia group in both timed and untimed tests of word reading and decoding skills), or history of developmental dyslexia who were matched to the dyslexia group on age and cognitive ability, as measured by the Raven Matrices tests (Raven, Court, \& Raven, 1992). Based on these criteria, one participant with dyslexia was excluded from the analysis. Written informed consent was obtained from all participants and they were compensated for their participation. The Institutional Review Board of Carnegie Mellon University approved the study and it was conducted in accordance with the Declaration of Helsinki.

All participants performed a battery of linguistic and cognitive tests to evaluate general cognitive ability, verbal working memory, rapid automatized naming reading skills, and phonological awareness. Details about these tasks are presented in Table 1 . These tests were administered to confirm that groups differed significantly in phonological and reading skills, with similar levels of intelligence.

Indeed, the groups did not differ according to age or intelligence (Table 2). However, compared with the normal reading group, the dyslexia group showed a clear profile of reading disability, conforming to the symptomatology of dyslexia. The group differed significantly from the control group on word reading and decoding skills, in both rate and accuracy measures. In addition, the dyslexia group exhibited characteristic deficits in the three major phonological domains: phonological awareness (Spoonerisms), verbal short-term memory (digit span), and rapid naming (rapid automatized naming).

Notably, the dyslexia group was comprised of highfunctioning university students with dyslexia. Such highfunctioning adults typically exhibit average performance on standardized reading tests (including performance on reading low frequency words, as in the Woodcock Reading Mastery Test-Revised (Wilson \& Lesaux, 2001). Nevertheless, the demands of complex phonological tests such as the Spoonerism test can identify highly compensated adults with a history of developmental dyslexia (Wilson \& Lesaux, 2001). Our dyslexic participants match this profile. All participants in the dyslexia group had a history of developmental dyslexia identified in childhood development by a qualified psychologist. Furthermore, all were university students receiving testing accommodations. Despite performance with in the normal range on standardized reading tests, these participants performed significantly more poorly than matched controls on all literacy measures and exhibited phonological processing impairments under high phonological demands (as in the Spoonerism test). This profile is clearly indicative of a sample of dyslexic adults.

\section{Stimuli}

The procedure and stimulus design followed Guediche et al. (2016, Experiment 3). Stimuli were drawn from seven 50-word phonetically balanced English monosyllabic word lists (Egan, 1948; list 1-7). Words were monosyllabic and high frequency, and therefore relatively easy to read. Words were spoken by a female monolingual English talker (LLH), recorded using a digital Marantz PMC670 recorder (16-bit, $22050 \mathrm{~Hz}$ ), matched in root-mean-square amplitude and saved as a digital file.

We used a signal processing technique from the cochlear implant literature to introduce an incremental signal distortion that systematically influenced speech intelligibility (Shannon, Zeng, Kamath, Wygonski, \& Ekelid, 1995; Zeng, 2004). The resulting vocoded, spectrally shifted speech introduced distortion to the signal by vocoding natural speech into 20 bands, limiting the spectral resolution of available acoustic speech information. We also introduced a spectral shift between the filters applied to analysis of the speech input and the output filters to which input is passed to create a spectral shift in the final distorted speech signal. With increasingly large disparities in the spectral shift between input and output filters, speech intelligibility decreases (Guediche et al., 2016).

Distortions to the natural speech stimuli were created using Tiger Speech software (see Fu \& Galvin, 2003). 
Table 1. Psychometric tests

The following tests were administered according to the test manual instructions:

1. Raven's Standard Progressive Matrices test (Raven et al., 1992) - nonverbal intelligence was assessed by the Raven's Progressive Matrices test. This task requires participants to choose the item from the bottom of the figure that would complete the pattern at the top. The maximum raw score is 60 . Test reliability coefficient is .9

2. Digit span from the Wechsler Adult Intelligence Scale (WAIS-III; Wechsler, 1997) - in this task, participants are required to recall the names of the digits presented auditorily in the order they appeared with a maximum of total raw score of 28 . Task administration is discontinued after a failure to recall two trials with a similar length of digits. Test reliability coefficient is .9

3. Rapid Automatized Naming (Denckla \& Rudel, 1976) - the tasks require oral naming of rows of visually presented exemplars drawn from a constant category (RAN colors, RAN categories, RAN numerals, and RAN letters). It requires not only the retrieval of a familiar phonological code for each stimulus, but also coordination of phonological and visual (color) or orthographic (alphanumeric) information quickly in time. The reliability coefficient of these tests ranging between .98 and .99 .

4. Woodcock Reading Mastery Test Word Identification and Word Attack subtests (Woodcock, 1987). The Word Identification subtest measures participants' ability to accurately pronounce printed English words, ranging from high to low frequency of word occurrence with a maximum of total raw score of 106 . Test reliability coefficient is .97. The Word Attack subtest assesses participants' ability to read pronounceable nonwords varying in complexity with a maximum total raw score of 45 . Test reliability coefficient is .87. Task administration is discontinued when six consecutive words are read incorrectly.

5. Sight Word Efficiency (i.e., rate of word identification) and Phonemic Decoding Efficiency (i.e., rate of decoding pseudowords), and subtests from the Test of Word Reading Efficiency (TOWRE-II; Torgesen et al., 1999) were used to measure reading rate. The test contains two timed measures of real-word reading and pseudoword decoding. Participants are required to read the words aloud as quickly and accurately as possible. The score reflects the total number of words/nonwords read correctly in a fixed 45-s interval. Task administration is discontinued after $45 \mathrm{~s}$. Sight word efficiency maximum raw score is 108 . Phonemic decoding efficiency maximum raw core is 65 . Test-retest reliability coefficients for these subtests are .91 and .90 , respectively.

6. The Nelson-Denny Vocabulary Test (Nelson, Denny, \& Brown, 1960). The test provides a vocabulary measure. It contains 100 sentences for which the subject has to circle the right word from 5 response options with words drawn from high school and college textbooks and vary in difficulty. Number of accurate responses was calculated. Maximum raw score is 100.

7. Spoonerism Test (adapted from Brunswick et al., 1999) - the test provides a measure of phonemic segmentation skill. Participants are required to reverse initial phoneme of words to artificially produce a type of Spoonerism that takes place in spontaneous speech and that provides evidence for the phoneme as a linguistic unit. For example, the word pair "Basket Lemon" becomes "Lasket Bemon". The maximum raw score is 12 .

Table 2. Demographic and psychometric data of dyslexia and control groups

\begin{tabular}{|c|c|c|c|c|c|c|}
\hline \multirow[b]{2}{*}{ Measure } & \multicolumn{6}{|c|}{ Group } \\
\hline & Dyslexia mean (SD) & Range & Control mean (SD) & Range & $\mathrm{P}$ & Cohen's d \\
\hline Age (in years) & $22(4.9)$ & $18-35$ & $22(3.5)$ & $18-30$ & n.s. & 0 \\
\hline Raven's SPM & $56.87(2.87)$ & $51-60$ & $57.05(2.51)$ & $52-60$ & n.s. & .06 \\
\hline Digit $\operatorname{span}^{\mathrm{a}}$ (combined) & $10.31(2.86)$ & $5-16$ & $13.7(3.45)$ & 6-19 & $<.01$ & 1.06 \\
\hline RAN objects ${ }^{\mathrm{a}}$ & $102.12(18.61)$ & $74-129$ & $115.76(12.58)$ & $93-133$ & $<.001$ & .85 \\
\hline RAN colors ${ }^{\mathrm{a}}$ & $96.62(11.92)$ & $80-118$ & $111(6.91)$ & $97-124$ & $<.001$ & 1.47 \\
\hline RAN numbers ${ }^{\mathrm{a}}$ & $105.87(5.35)$ & $95-113$ & $113.76(3.68)$ & $109-120$ & $<.001$ & 1.71 \\
\hline RAN letters ${ }^{\mathrm{a}}$ & $102.12(5.94)$ & $85-111$ & $111.11(4.55)$ & $102-117$ & $<.001$ & 1.69 \\
\hline WRMT-R WI ${ }^{\mathrm{a}}$ & $98(3.94)$ & $92-107$ & $109.05(6.45)$ & $100-126$ & $<.001$ & 2.06 \\
\hline WRMT-R WA ${ }^{\mathrm{a}}$ & $95.31(7.48)$ & $82-110$ & $113.94(11.21)$ & $100-134$ & $<.001$ & 1.95 \\
\hline TOWRE SW $(\mathrm{A}+\mathrm{B})^{\mathrm{a}}$ & $96.43(7.95)$ & $81-112$ & $116.35(7.8)$ & $101-127$ & $<.001$ & 2.52 \\
\hline TOWRE PD $(A+B)^{\mathrm{a}}$ & $88.87(7.12)$ & 72-98 & $114.41(10.48)$ & $100-127$ & $<.001$ & 2.85 \\
\hline Spoonerism time & $140.5(78.2)$ & $82-368$ & $98(32.51)$ & $63-156$ & $<.05$ & .71 \\
\hline Spoonerism accuracy & $7.93(3.53)$ & $1-12$ & $10.88(2.11)$ & $4-12$ & $<.01$ & 1.01 \\
\hline Nelson-Denny Test (vocabulary subtest) & $81.13(11.08)$ & $60-95$ & $88.18(8.34)$ & $72-96$ & $=.053$ & .71 \\
\hline
\end{tabular}

${ }^{\text {a }}$ Standard scores (whereby smaller numbers are expected for dyslexia group), other scores are raw scores. RAN $=$ Rapid Naming, WI $=$ Word Identification, $\mathrm{WA}=$ Word Attack, SA = Sight Word, PD = Phonological Decoding.

Each spoken word was band-pass filtered into 20 frequency bands using eighth-order Butterworth filters with $24 \mathrm{~dB} /$ octave falloff. The frequency band cut-offs were chosen on the basis of the Nucleus-24M cochlear implant (covering frequencies from 116 to $7871 \mathrm{~Hz}$ ). The temporal envelope of each band was removed by half-wave rectification and 


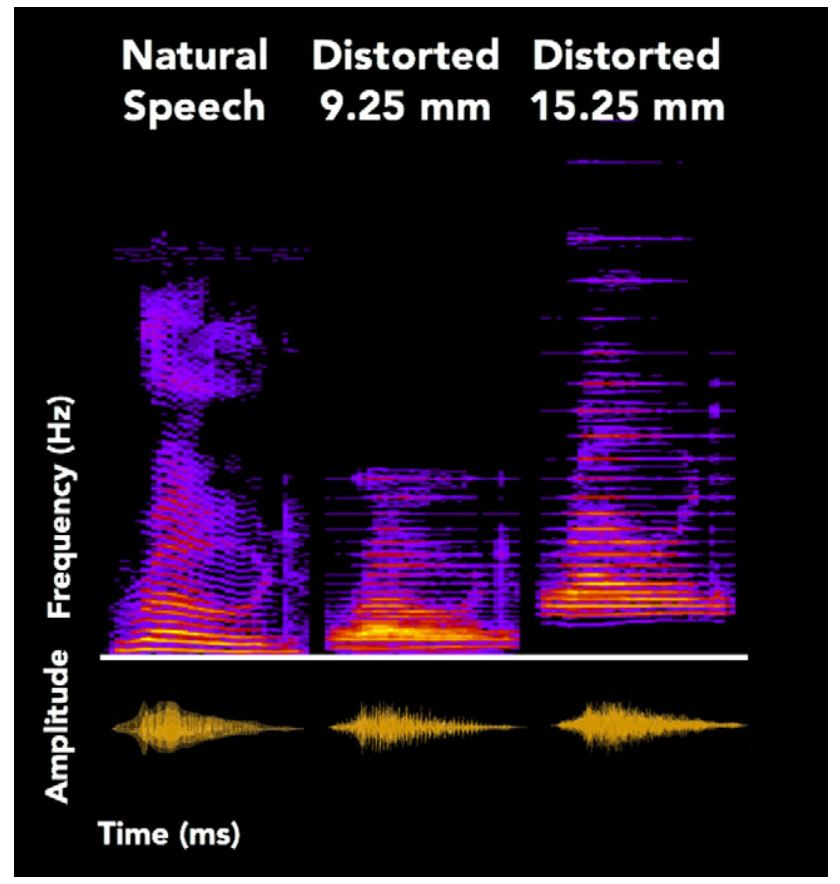

Fig. 1. Example of waveforms (amplitude as a function of time, bottom) and spectrograms (frequency as a function of time, with hot colors illustrating greater amplitudes, top) for natural undistorted speech (left), a mild signal distortion (middle, $9.25 \mathrm{~mm}$ distortion), and a severe signal distortion (right, $15.25 \mathrm{~mm}$ distortion). Each was created from a natural production of the word road.

low-pass filtering at $160 \mathrm{~Hz}$. This envelope was created in order to modulate a carrier band. We used the following equation in order to compute the frequency range of the carrier band. Here, $P 0$ is the "insertion depth" (in millimeters) of the theoretical implant model, with $i$ corresponding to the 20 frequency bands:

$$
p(i)=P 0+0.75 * i, i=0,1,2,3 \ldots 20
$$

The carrier band frequencies were computed with the assumption of a 35-mm cochlea (Greenwood, 1990) and were determined by:

$$
f(i)=165.4 *(10 p(i) * 0.06-0.88)
$$

Together these two equations defined the corner frequencies of the carrier bands, calculated to generate the spectrally shifted speech tokens across increasing "insertion depths" corresponding to greater spectral shifts. The most apical carrier band location $(P 0)$ ranged between 9.25 and $15.25 \mathrm{~mm}$ from the apex of the cochlea. This created a range of $9.25 \mathrm{~mm}$ to $15.25 \mathrm{~mm}$ insertion depths and shifted the vocoded speech spectra incrementally upward through the frequency dimension, as illustrated in Figure 1. Carrier bands incrementing in steps of $0.25 \mathrm{~mm}$ insertion depth were generated between these endpoint values. For example, no spectral energy below $448 \mathrm{~Hz}$ was present for an insertion depth of
$9.25 \mathrm{~mm}$ speech in which speech was shifted upward in frequency (lowest distortion). For the highest distortion $(15.25 \mathrm{~mm})$, there was no spectral energy below $1214 \mathrm{~Hz}$. Crossover reduction between neighboring bands was $-3 \mathrm{~dB}$.

\section{Procedure}

Participants were seated in a sound-attenuated booth with a computer monitor mounted at eye level. Stimulus presentation and the recording of response time and accuracy were controlled by a computer program (E-prime; Schneider, Eschman, \& Zuccolotto, 2002). On each trial, participants heard a distorted spoken word diotically over headphones (Beyer DT-150, approximately $70 \mathrm{~dB}$ ) and typed what they heard using a keyboard within a 4-s time window. Participants were encouraged to guess if they were unsure of the word. The typed response appeared on the screen and participants were allowed to use backspace to correct typing errors. Participants indicated that their response was complete with a key press. Immediately thereafter, they heard the distorted word repeated with concurrent presentation of the visual orthographic representation of the word as feedback. The visual orthographic feedback was presented for a length of $1000 \mathrm{~ms}$ thereby assuring that even participants with slow reading skills would receive and comprehend the orthographic feedback.

The task involved a pretest, training, and a posttest which required open-set speech recognition (i.e., participants freely typed each word they heard, with no response options provided). Pre- and posttests each involved 50 unique words that were not previously encountered in the task, presented at the highest level of distortion (15.25 mm insertion depth) that, in the Guediche et al. (2016) study, resulted in open-set speech recognition of about $10 \%$ correct. No orthographic feedback was provided in the pre- and posttests. The pretest established baseline word recognition performance for a severe distortion and posttest performance provided a measure of the improvement in speech recognition relative to baseline, as a function of the adaptive plasticity evoked in the training block.

Following the approach of Guediche et al. (2016, Experiment 3), the training block involved both incremental increases in speech distortion and orthographic feedback. At the commencement of training, words with modest distortion $(9.25 \mathrm{~mm}$ insertion depth, approximately $55 \%$ correct in open-set speech recognition in Guediche et al., 2016) were presented. After each 10 trials, regardless of performance, the speech signal distortion was increased by incrementing the spectral shift $(+0.25 \mathrm{~mm}$ insertion depth). This resulted in 25 distortion increments across 250 trials of training. All words presented in training were unique and distinct from those presented at pre- and posttests.

Across pretest, training, and posttest phases of the study, responses were scored as correct if they phonologically matched the undistorted spoken word. Misspellings were not coded as mistakes. The first author and a research 
assistant checked each response several times to assure misspellings were not coded as error for both dyslexia and control groups. The percent of trials with observed misspellings was relatively small $3.3 \%$ in the group and $3 \%$ in the control group). The experiment was conducted across two sessions. Participants performed the cognitive and linguistics tests first in a 1 -hr session. In a second 20-min session, participants completed the word recognition task across pretest, training, and posttest blocks.

\section{RESULTS}

\section{Data Analysis}

Participants' performance during pretest, posttest, and training phases was calculated as the mean proportion of words correctly recognized across all words in a given phase.

We first calculated participants' level of performance during the pretest phase as a measure of baseline word recognition performance for severely distorted speech. We next divided the training phase into 10-trial bins to assess word recognition across training. We measured generalization of training to novel words not experienced in training by comparing word recognition performance at posttest relative to pretest. Finally, we examined the correlation between word recognition performance in training and word recognition improvement from pretest to posttest as a means of investigating the extent to which internal predictions from accurate lexical mapping might relate to the degree of adaptive plasticity observed across groups.

\section{Word Recognition at Pretest}

As shown in Figure 2, word recognition across the 50 highly distorted speech signals (15.25 mm insertion depth) experienced at pretest was very poor. On average, the control group accurately recognized an average of 6.52 words out of 50 $(\mathrm{SE}=8.2)$ for $13.05 \%$ average accuracy. The dyslexia group accurately recognized an average of 4.6 of the 50 severely distorted words $(S D=6.4)$ for $9.2 \%$ average accuracy. The two groups did not significantly differ in pretest word comprehension, as reflected by an independent samples $t$-test, $t(1,31)=-1.47, p=.15$, Cohen's $\mathrm{d}=.56$.

\section{Word Recognition in Training}

Figure 3 plots average word recognition accuracy as a function of the severity of the speech distortion across training for each group. During training, the signal distortion began at a moderate level and incremented each 10 trials, with orthographic feedback provided on each of the 250 trials. We examined word recognition accuracy as a function of the speech signal distortion, which increased every 10 trials, with a mixed-model ANOVA with Group as a between-subjects factor and signal distortion as a within-subjects factor. This revealed a significant main effect of Group, $F(1,31)=$

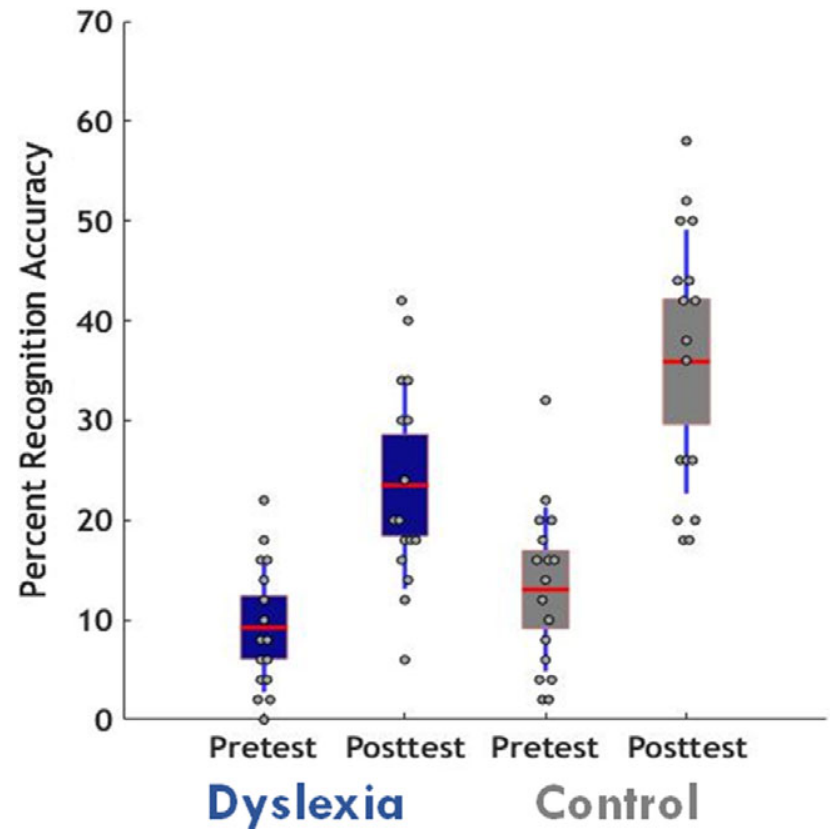

Fig. 2. Average percent speech recognition accuracy across the most severe speech distortion at pretest and posttest for dyslexia and control groups. Each word presented in the experiment was unique, meaning that posttest gains reflect generalization of adaptive plasticity arising from the intervening training block. Data points represent individual participants, the red bar indicates the mean, and error bars represent one standard error of the mean.

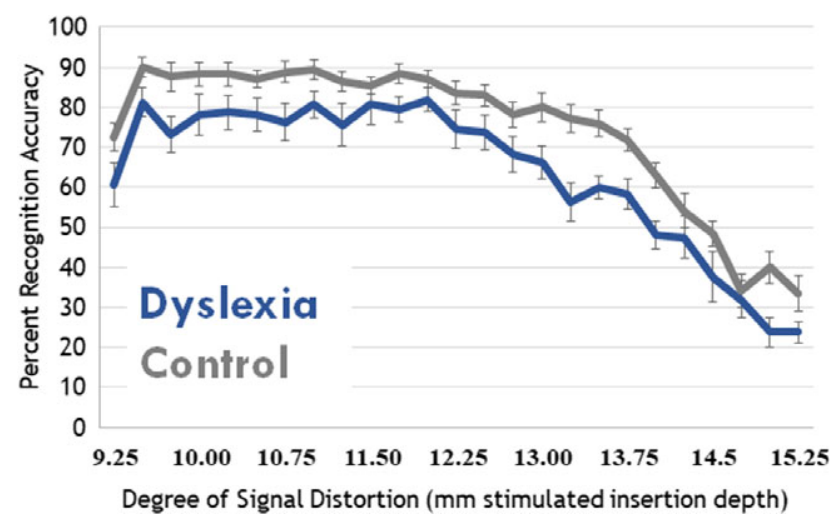

Fig. 3. Average percent speech recognition accuracy across 250 training trials for which the signal distortion began at a moderate level (simulated $9.25 \mathrm{~mm}$ insertion depth) and incremented each 10 trials, with orthographic feedback provided on each trial. Data are divided into 25 10-word epochs, each corresponding to an increment in the signal distortion (simulated $+0.25 \mathrm{~mm}$ insertion depth). Error bars represents one standard error of the mean.

17.59, $p=.001, \eta_{p}{ }^{2}=.35$, as well as a main effect of the increasing signal distortion across training, $F(24,744)=$ $58.40, p=.001, \eta_{p}{ }^{2}=.65$. There was no interaction between these factors, $F(24,744)=.71, p=.841 ; \eta_{p}^{2}=.02$. Across training, individuals in the dyslexia group recognized significantly fewer words $(M=63 \%, S E=1 \%)$ than listeners in the control group $(M=74 \%, S . E .=1 \%)$. 


\section{Word Recognition at Posttest}

Word recognition at posttest provides a measure of the generalization of adaptive plasticity evoked in the training block because each word presented in the experiment is unique. Any pre to posttest improvement in word recognition for the most severely distorted speech must arise as a result of generalization (e.g., Schwab et al., 1985; Guediche et al., 2016). Given that there was no baseline group difference in performance at pretest, we evaluated adaptive plasticity with a $2 \times 2$ mixed-model ANOVA with testing phase (pretest and posttest), as a within-subjects factor and Group (dyslexia, control) as a between-subjects factor. There was a significant main effect of testing phase, $F(1,31)=105.8$, $p=.00000, \eta_{p}{ }^{2}=.77$, such that there was a general improvement in word recognition for severely distorted speech from pre- to posttest $(M=11 \%, S . E .=1.29, M=20 \%, S . E .=2.24$, for pre $v s$. posttest, respectively) consistent with adaptive plasticity. There also was a main effect of group, $F(1,31)=7.4, p=.010, \eta_{p}{ }^{2}=.15$. In general, across preand posttests, individuals with dyslexia were less accurate $(M=16 \%, S . E .=.21)$ in word recognition than control listeners $(M=24.20 \%$, S.E. $=.20)$. Crucially, these main effects must be understood in the context of a significant group by testing phase interaction, $F(1,31)=5.7, p=.023, \eta_{p}{ }^{2}=$ .15. Whereas there were no significant group differences in performance at pretest, $t(1,31)=-1.47, p=.15$, Cohen's $\mathrm{d}=.56$ the control group $(M=35.88 \%$, S.E. $=1.32 \%)$ outperformed individuals with dyslexia $(M=23.5 \%$, $S D=1.03 \%)$ at posttest, $t(1,31)=-2.97, p=.005$, Cohen's $d=1.03$. Adaptive plasticity drove a $22.83 \%$ improvement in word recognition accuracy among the control group, but only a $14.8 \%$ improvement among the dyslexia group.

In order the test the possibility that differences in vocabulary knowledge influence adaptive plasticity, we conducted a $2 \times 2$ mixed-model ANCOVA with testing phase (pretest and posttest), as a within-subjects factor and Group (dyslexia, control) as a between-subjects factor and vocabulary score (Nelson-Deny test, see Table 1) as a covariate. The interaction of testing phase and group was still significant, $F(1,29)=5.88, p=.022 ; \eta_{p}{ }^{2}=.71$, suggesting that differences in accessing lexical knowledge could not fully account for the observed differences in adaptive plasticity across groups.

\section{Relationship of Training Performance with Generalization of Learning Gains}

Guediche et al. (2016) reported a correlation between the magnitude of adaptive plasticity observed for an individual listener (posttest minus pretest accuracy in recognizing highly distorted speech) and overall word recognition accuracy in the training block. Here, as shown in Figure 4, we observe a modest correlation of roughly the same magnitude as Guediche et al. for data pooled across the groups, $r(33)=.376, p=.015$. More accurate recognition of distorted words during training resulted in the greatest adaptive

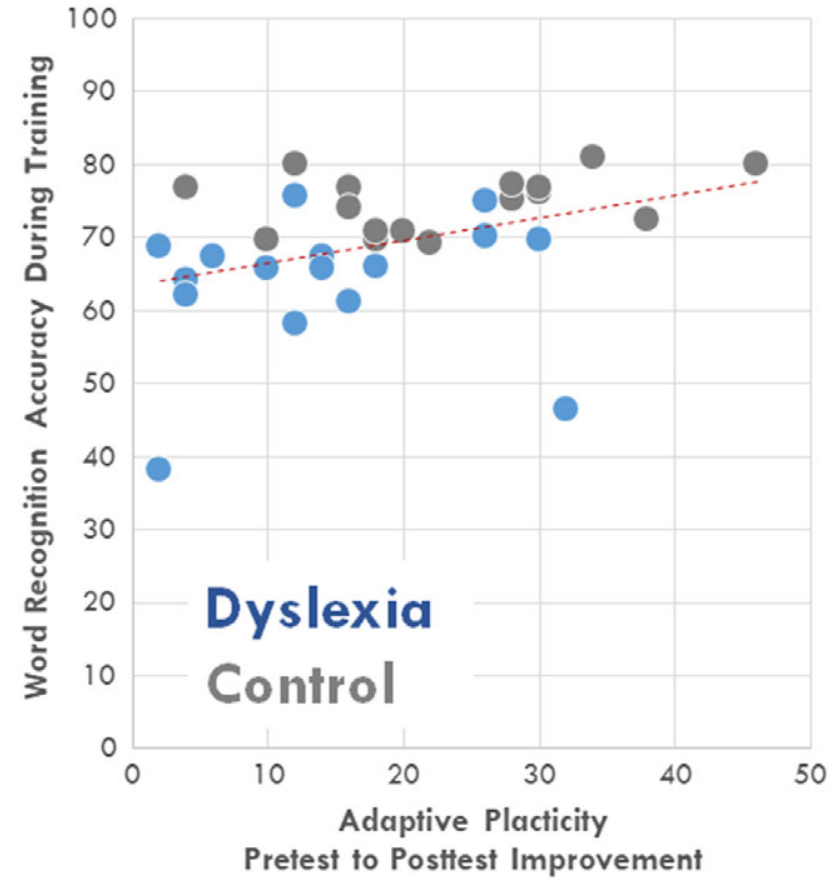

Fig. 4. The magnitude of the adaptive plasticity effect (posttest minus pretest word recognition) as a function of the accuracy of word recognition during training for all participants (individuals in the dyslexia group are plotted in blue, those in the control group are plotted in gray).

plasticity. This is consistent with the notion that internally generated predictions available when distorted input can be successfully mapped to word knowledge play a role in adaptive plasticity (Guediche et al., 2016).

\section{GENERAL DISCUSSION}

In the present study, individuals with dyslexia exhibited less adaptive plasticity than age and intelligence matched peers. Posttest baseline speech recognition for severely distorted speech was very poor for both the dyslexia and control groups, with no significant difference at pretest. Yet, across the full course of 250 training trials in which the signal distortion increased incrementally every 10 trials, the control group was significantly more accurate at word recognition than the dyslexic group. At posttest, recognition of the most severe speech distortion had improved by $22.8 \%$ relative to pretest, whereas the dyslexia group had improved by just $14.8 \%$. The dyslexia group exhibited significantly less adaptive plasticity in word recognition than the control group.

Prior results with typical listeners performing this same task have demonstrated that when speech input is moderately distorted and word recognition is therefore somewhat accurate, even mere exposure can be sufficient to drive adaptive plasticity. But, when speech input is so severely distorted that it no longer provides sufficient access to internal predictions through, for example, access to lexical representations that guide accurate word recognition adaptive plasticity can be driven by external (e.g., orthographic feedback) information 
sources that link the distorted input to internal representations (Guediche et al., 2016). In this prior work, and also here among control and dyslexia groups, the accuracy of linguistic predictions as measured by word recognition accuracy was related to the degree of adaptive plasticity observed. The ability to map distorted speech input to internal, here lexical, representations is associated with the degree of adaptive plasticity observed. Guediche et al. $(2014,2015)$ propose that when disambiguating information is available with distorted speech input - such as that evoked by activation of an internal linguistic representation (Guediche et al., 2016) - a mismatch or "conflict" between the predicted and actual input may generate an internal error signal that may, in turn, guide adaptive plasticity through supervised error-driven learning. Guediche et al. (2016) provide evidence that internal lexical predictions may drive this form of procedural learning and that it may involve the cerebellum (Guediche et al., 2015).

From this perspective, disorders that impact the fidelity of the linguistic representations that provide the internal predictions hypothesized to drive adaptive plasticity (or the learning mechanisms that make use of these predictions to adjust the mapping of distorted speech input) would be expected to impact the degree of adaptive plasticity observed in speech recognition. In this regard, evidence indicating that individuals with developmental dyslexia exhibit impairments in the fidelity of speech processing (Kast, Elmer, Jancke, \& Meyer, 2010) and in vocabulary size (van Viersen et al., 2017), and parallel literatures demonstrating procedural learning impairments (Gabay et al., 2012a, 2012c; Gabay, Vakil, et al., 2015; Hedenius et al., 2013; Howard et al., 2006; Lum et al., 2013; Pavlidou \& Williams, 2014; Stoodley et al., 2008; Stoodley et al., 2006; Vicari et al., 2005) and neurobiological differences in the cerebellum (Fernandez et al., 2016; Stanberry et al., 2006; Stoodley, 2014), potentially involved in guiding adaptive plasticity in speech (Guediche et al., 2015), align with the present observation of less efficient learning in individuals with developmental dyslexia.

For example, the more limited vocabularies among the dyslexia group (Table 2) might affect the fidelity of linguistic representations, and thus adaptive plasticity in speech perception. Greater vocabulary knowledge likely supports ready word recognition from unfamiliar or ambiguous auditory input (Borovsky, Elman, \& Fernald, 2012). One may argue, therefore, that poorer vocabularies among the dyslexia group may have made it less possible to make use of word knowledge to generate internal predictions to drive adaptive plasticity. However, this explanation is weakened by evidence that there were no group differences in word recognition for the most severely distorted speech at pretest. Furthermore, group differences remained when variability derived from an estimate of vocabulary (the Nelson-Denny test) were factored into analyses.

Problems with reading and writing could have influenced the ability of people with dyslexia to perform the task. However, all stimuli were high-frequency monosyllabic English words, the duration of orthographic feedback was long, and response accuracy coding as done in such a way that misspellings were not considered errors. Similarly, poorer auditory or phonetic fidelity of speech processing in dyslexia (Kast et al., 2010) may have played a role in the less efficient adaptive plasticity observed among the dyslexic group. However, these representational challenges would be expected to influence participants' performance at baseline pretest, as well. The observation of no group differences at baseline reduces the possibility that problems in speech sound representations can account for the findings.

In fact, group differences emerged in training. In light of evidence that individuals with developmental dyslexia exhibit less efficient procedural learning across a variety of domains (Gabay et al., 2012a, 2012c; Gabay, Vakil, et al., 2015; Howard et al., 2006; Lum et al., 2013; Stoodley et al., 2008; Stoodley et al., 2006; Vicari et al., 2005), it is possible that the present results may reflect procedural-learning-related group differences. Gabay and Holt (2015) have argued that procedural learning impairment in dyslexia may lead to impaired perceptual learning that results in impoverished representations of the phonological characteristics of speech and concomitant difficulties in grapheme-phenome conversion and in learning to read (see also, Krishnan, Watkins, \& Bishop, 2016; Ullman et al., 2020). Therefore, it is possible that an impairment or inefficiency of the procedural learning systems could influence phonological processing by a perceptual route in addition the motor route originally suggested by Nicolson and Fawcett (Nicolson \& Fawcett, 2011). Indeed, there is emerging evidence that individuals with dyslexia may acquire sound categories through procedural learning less efficiently than typical listeners (Gabay \& Holt, 2015; Lim, Fiez, \& Holt, 2019).

The present results suggest the possibility that individuals with dyslexia also may exhibit less efficient adaptation across existing phonological representations in the context of online listening. As reviewed briefly above, speech processing is remarkably dynamic and adjusts rapidly to the mapping of acoustic speech input to linguistically relevant representations in online speech processing (Bertelson, Vroomen, \& De Gelder, 2003; Bradlow \& Bent, 2008; Idemaru \& Holt, 2011; Norris et al., 2003; Samuel \& Kraljic, 2009; Schwab et al., 1985; Zhang \& Holt, 2018). Although the mechanisms driving these adaptive plasticity effects are still unknown, multiple groups have proposed supervised error-driven learning as a possibility (Guediche, 2017; Guediche et al., 2014; Norris et al., 2003; Vroomen et al., 2007) and at least one study has implicated the cerebellum as a key component of the neurobiological network that is involved (Guediche et al., 2015). Thus, adaptive plasticity may represent another form of procedural learning postulated by Nicolson and Fawcett (2011) to be impaired in developmental dyslexia.

In line with this possibility, Gabay, Karni, and Banai (2018) recently observed that individuals with developmental dyslexia were on par with control participants in a task involving adaptive plasticity to time-compressed speech so 
long as stimuli were repeated across the task. When the potential for reliance on declarative memorization strategies was eliminated by introducing entirely novel speech stimuli (as in the present experiment), individuals with dyslexia exhibited significantly less adaptive plasticity than control listeners.

The present results indicate that even well-compensated adults with a history of dyslexia exhibit inefficiencies in adaptive plasticity in speech processing. Less robust adaptive plasticity in speech perception may affect those with dyslexia and, in turn, their speech comprehension abilities in realworld listening environments. Further research examining this and other phenomena of adaptive plasticity among individuals with dyslexia will be informative in revealing the nature of speech processing in dyslexia and, perhaps, in revealing mechanisms of adaptive plasticity among typical listeners, as well.

\section{ACKNOWLEDGMENTS}

Research was supported by the National Institutes of Health (R01DC004674) to LLH and by Binational Scientific Foundation (2015227) and the National Science Foundation-Binational Scientific Foundation (2016867) grants to LLH and YG. Thanks to Christi Gomez for stimulus creation and support in testing human participants.

\section{CONFLICT OF INTEREST}

The authors have nothing to disclose.

\section{REFERENCES}

Altmann, G.T., \& Young, D. (1993). Factors affecting adaptation to time-compressed speech. Paper presented at the Third European Conference on Speech Communication and Technology.

Alvarez, T.A., \& Fiez, J.A. (2018). Current perspectives on the cerebellum and reading development. Neuroscience \& Biobehavioral Reviews, 92, 55-66.

Banai, K., \& Lavner, Y. (2012). Perceptual learning of timecompressed speech: more than rapid adaptation. PloS One, 7(10), e47099.

Banai, K., \& Lavner, Y. (2014). The effects of training length on the perceptual learning of time-compressed speech and its generalization. The Journal of the Acoustical Society of America, 136(4), 1908-1917.

Bertelson, P., Vroomen, J., \& De Gelder, B. (2003). Visual recalibration of auditory speech identification: a McGurk aftereffect. Psychological Science, 14(6), 592-597.

Borovsky, A., Elman, J.L., \& Fernald, A. (2012). Knowing a lot for one's age: vocabulary skill and not age is associated with anticipatory incremental sentence interpretation in children and adults. Journal of Experimental Child Psychology, 112(4), 417-436.

Bradlow, A.R., \& Bent, T. (2008). Perceptual adaptation to nonnative speech. Cognition, 106(2), 707-729.

Brookes, R.L., Nicolson, R.I., \& Fawcett, A.J. (2007). Prisms throw light on developmental disorders. Neuropsychologia, 45(8), 1921-1930.
Brunswick, N., McCrory, E., Price, C., Frith, C., \& Frith, U. (1999). Explicit and implicit processing of words and pseudowords by adult developmental dyslexics: a search for Wernicke's Wortschatz? Brain, 122(10), 1901-1917.

Cainer, K.E., James, C., \& Rajan, R. (2008). Learning speech-innoise discrimination in adult humans. Hearing research, 238(1-2), 155-164.

Clarke, C.M., \& Garrett, M.F. (2004). Rapid adaptation to foreignaccented english. The Journal of the Acoustical Society of America, 116(6), 3647-3658.

Cohen-Mimran, R., \& Sapir, S. (2007). Auditory temporal processing deficits in children with reading disabilities. Dyslexia, 13(3), 175-192.

Cooper, A., Brouwer, S., \& Bradlow, A. R. (2015). Interdependent processing and encoding of speech and concurrent background noise. Attention, Perception, \& Psychophysics, 77(4), 1342-1357.

Davis, M.H., Johnsrude, I.S., Hervais-Adelman, A., Taylor, K., \& McGettigan, C. (2005). Lexical information drives perceptual learning of distorted speech: evidence from the comprehension of noise-vocoded sentences. Journal of Experimental Psychology: General, 134(2), 222.

Denckla, M.B., \& Rudel, R.G. (1976). Rapid 'automatized' naming (R.A.N.): Dyslexia differentiated from other learning disabilities. Neuropsychologia, 14, 471-479.

Egan, J.P. (1948). Articulation testing methods. The Laryngoscope, 58(9), 955-991.

Farmer, M.E., \& Klein, R.M. (1995). The evidence for a temporal processing deficit linked to dyslexia: a review. Psychonomic Bulletin \& Review, 2(4), 460-493.

Fawcett, A.J., \& Nicolson, R.I. (2008). Dyslexia and the Cerebellum. In G. Reid, A. Fawcett, F. Manis, \& L. Siegel (Eds.), The sage handbook of dyslexia, (pp. 77-98). London: Sage.

Fernandez, V.G., Juranek, J., Romanowska-Pawliczek, A., Stuebing, K., Williams, V.J., \& Fletcher, J.M. (2016). White matter integrity of cerebellar-cortical tracts in reading impaired children: a probabilistic tractography study. Brain and Language, 161, 45-56.

Francis, A.L., Baldwin, K., \& Nusbaum, H.C. (2000). Effects of training on attention to acoustic cues. Perception \& psychophysics, 62(8), 1668-1680.

Francis, A.L., Nusbaum, H.C., \& Fenn, K. (2007). Effects of training on the acoustic-phonetic representation of synthetic speech. Journal of Speech, Language, and Hearing Research, 50, 1445-1465.

Fu, Q.-J., \& Galvin III J.J. (2003). The effects of short-term training for spectrally mismatched noise-band speech. The Journal of the Acoustical Society of America, 113(2), 1065-1072.

Gabay, Y., \& Holt, L.L. (2015). Incidental learning of sound categories is impaired in developmental dyslexia. Cortex, 73, 131-143.

Gabay, Y., Karni, A., \& Banai, K. (2017). The perceptual learning of time-compressed speech: a comparison of training protocols with different levels of difficulty. PloS One, 12(5), e0176488.

Gabay, Y., Karni, A., \& Banai, K. (2018). Learning to decipher timecompressed speech: Robust acquisition with a slight difficulty in generalization among young adults with developmental dyslexia. PloS one, 13(10), e0205110.

Gabay, Y., Najjar, I.J., \& Reinisch, E. (2019). Another temporal processing deficit in individuals with developmental dyslexia: The case of normalization for speaking rate. Journal of Speech, Language, and Hearing Research, 62(7), 2171-2184.

Gabay, Y., Schiff, R., \& Vakil, E. (2012a). Attentional requirements during acquisition and consolidation of a skill in normal readers and developmental dyslexics. Neuropsychology, 26(6), 744. 
Gabay, Y., Schiff, R., \& Vakil, E. (2012b). Dissociation between online and offline learning in developmental dyslexia. Journal of Clinical and Experimental Neuropsychology, 34(3), 279-288.

Gabay, Y., Schiff, R., \& Vakil, E. (2012c). Dissociation between the procedural learning of letter names and motor sequences in developmental dyslexia. Neuropsychologia, 50(10), 2435-2441.

Gabay, Y., Thiessen, E.D., \& Holt, L.L. (2015). Impaired statistical learning in developmental dyslexia. Journal of Speech, Language, and Hearing Research, 58(3), 934-945.

Gabay, Y., Vakil, E., Schiff, R., \& Holt, L. L. (2015). Probabilistic category learning in developmental dyslexia: evidence from feedback and paired-associate weather prediction tasks. Neuropsychology, 29(6), 844.

Greenspan, S.L., Nusbaum, H.C., \& Pisoni, D.B. (1988). Perceptual learning of synthetic speech produced by rule. Journal of Experimental Psychology: Learning, Memory, and Cognition, 14(3), 421.

Greenwood, D.D. (1990). A cochlear frequency-position function for several species-29 years later. The Journal of the Acoustical Society of America, 87(6), 2592-2605.

Guediche, S. (2017). Flexible and adaptive processes in speech perception. The Speech Processing Lexicon: Neurocognitive and Behavioural Approaches, 22, 155.

Guediche, S., Blumstein, S., Fiez, J., \& Holt, L.L. (2014). Speech perception under adverse conditions: insights from behavioral, computational, and neuroscience research. Frontiers in Systems Neuroscience, 7, 126.

Guediche, S., Fiez, J.A., \& Holt, L.L. (2016). Adaptive plasticity in speech perception: effects of external information and internal predictions. Journal of Experimental Psychology: Human Perception and Performance, 42(7), 1048.

Guediche, S., Holt, L.L., Laurent, P., Lim, S.-J., \& Fiez, J.A. (2015). Evidence for cerebellar contributions to adaptive plasticity in speech perception. Cerebral Cortex, 25(7), 1867-1877.

Hedenius, M., Persson, J., Alm, P.A., Ullman, M.T., Howard Jr, J.H., Howard, D.V., \& Jennische, M. (2013). Impaired implicit sequence learning in children with developmental dyslexia. Research in Developmental Disabilities, 34(11), 3924-3935.

Hervais-Adelman, A., Davis, M.H., Johnsrude, I.S., \& Carlyon, R.P. (2008). Perceptual learning of noise vocoded words: effects of feedback and lexicality. Journal of Experimental Psychology: Human Perception and Performance, 34(2), 460.

Holl, A.K., Wilkinson, L., Tabrizi, S.J., Painold, A., \& Jahanshahi, M. (2012). Probabilistic classification learning with corrective feedback is selectively impaired in early Huntington's disease evidence for the role of the striatum in learning with feedback. Neuropsychologia, 50(9), 2176-2186.

Howard Jr, J.H., Howard, D.V., Japikse, K.C., \& Eden, G.F. (2006). Dyslexics are impaired on implicit higher-order sequence learning, but not on implicit spatial context learning. Neuropsychologia, 44(7), 1131-1144.

Idemaru, K., \& Holt, L.L. (2011). Word recognition reflects dimension-based statistical learning. Journal of Experimental Psychology: Human Perception and Performance, 37(6), 1939.

Kalikow, D.N., Stevens, K.N., \& Elliott, L.L. (1977). Development of a test of speech intelligibility in noise using sentence materials with controlled word predictability. The Journal of the Acoustical Society of America, 61(5), 1337-1351.

Kast, M., Elmer, S., Jancke, L., \& Meyer, M. (2010). ERP differences of pre-lexical processing between dyslexic and nondyslexic children. International Journal of Psychophysiology, 77(1), 59-69.
Kita, Y., Yamamoto, H., Oba, K., Terasawa, Y., Moriguchi, Y., Uchiyama, H., ... Inagaki, M. (2013). Altered brain activity for phonological manipulation in dyslexic Japanese children. Brain, 136(12), 3696-3708.

Knowlton, B.J., Siegel, A.L., \& Moody, T.D. (2017). Procedural learning in humans. In J. H. Byrne (Ed.), Learning and memory: A comprehensive reference, (pp. 295-312). Oxford: Academic Press. doi:10.1016/B978-0-12-809324-5.21085-7

Krishnan, S., Watkins, K.E., \& Bishop, D.V. (2016). Neurobiological basis of language learning difficulties. Trends in Cognitive Sciences, 20(9), 701-714.

Lim, S.-J., Fiez, J.A., \& Holt, L.L. (2019). Role of the striatum in incidental learning of sound categories. Proceedings of the National Academy of Sciences, 116(10), 4671-4680.

Liss, J.M., Spitzer, S.M., Caviness, J.N., \& Adler, C. (2002). The effects of familiarization on intelligibility and lexical segmentation in hypokinetic and ataxic dysarthria. The Journal of the Acoustical Society of America, 112(6), 3022-3030.

Loebach, J.L., Pisoni, D.B., \& Svirsky, M.A. (2010). Effects of semantic context and feedback on perceptual learning of speech processed through an acoustic simulation of a cochlear implant. Journal of Experimental Psychology: Human Perception and Performance, 36(1), 224.

Lum, J.A., Ullman, M.T., \& Conti-Ramsden, G. (2013). Procedural learning is impaired in dyslexia: evidence from a meta-analysis of serial reaction time studies. Research in Developmental Disabilities, 34(10), 3460-3476.

Menghini, D., Hagberg, G.E., Caltagirone, C., Petrosini, L., \& Vicari, S. (2006). Implicit learning deficits in dyslexic adults: an fMRI study. NeuroImage, 33(4), 1218-1226.

Needle, J., Nicolson, R.I., \& Fawcett, A.J. (2015). Motor sequence learning in Dyslexia: is consolidation the key? BPA-Applied Psychology Bulletin (Bollettino di Psicologia Applicata), 63(273), 5-16.

Nelson, M.J., Denny, E.C., \& Brown, J.I. (1960). The Nelson-Denny Reading Test: Vocabulary-Com-prehension-Rate (Rev. ed.). New York: Hough-ton Mifflin.

Nicolson, R.I., Daum, I., Schugens, M.M., Fawcett, A.J., \& Schulz, A. (2002). Eyeblink conditioning indicates cerebellar abnormality in dyslexia. Experimental Brain Research, 143(1), 42-50.

Nicolson, R., \& Fawcett, A. (2010). Dyslexia, Learning, and the Brain. Cambridge, MA: MIT press.

Nicolson, R.I., \& Fawcett, A.J. (2011). Dyslexia, dysgraphia, procedural learning and the cerebellum. Cortex, 47(1), 117-127.

Nicolson, R.I., Fawcett, A.J., Berry, E.L., Jenkins, I.H., Dean, P., \& Brooks, D.J. (1999). Association of abnormal cerebellar activation with motor learning difficulties in dyslexic adults. The Lancet, 353(9165), 1662-1667.

Nicolson, R.I., Fawcett, A.J., \& Dean, P. (2001). Developmental dyslexia: the cerebellar deficit hypothesis. Trends in Neurosciences, 24(9), 508-511.

Norris, D., McQueen, J.M., \& Cutler, A. (2003). Perceptual learning in speech. Cognitive psychology, 47(2), 204-238.

Orban, P., Lungu, O., \& Doyon, J. (2008). Motor sequence learning and developmental dyslexia. Annals of the New York Academy of Sciences, 1145(1), 151-172.

Pallier, C., Sebastian-Gallés, N., Dupoux, E., Christophe, A., \& Mehler, J. (1998). Perceptual adjustment to time-compressed speech: a cross-linguistic study. Memory \& Cognition, 26(4), 844-851.

Paulesu, E., Frith, U., Snowling, M., Gallagher, A., Morton, J., Frackowiak, R.S., \& Frith, C.D. (1996). Is developmental 
dyslexia a disconnection syndrome? Evidence from PET scanning. Brain, 119(1), 143-157.

Pavlidou, E.V., \& Williams, J.M. (2014). Implicit learning and reading: insights from typical children and children with developmental dyslexia using the artificial grammar learning (AGL) paradigm. Research in Developmental Disabilities, 35(7), 1457-1472.

Pernet, C.R., Poline, J.B., Demonet, J.F., \& Rousselet, G.A. (2009). Brain classification reveals the right cerebellum as the best biomarker of dyslexia. BMC Neuroscience, 10(1), 67.

Petacchi, A., Laird, A.R., Fox, P.T., \& Bower, J.M. (2005). Cerebellum and auditory function: an ALE meta-analysis of functional neuroimaging studies. Human Brain Mapping, 25(1), 118-128.

Peterson, R.L., \& Pennington, B.F. (2015). Developmental dyslexia. Annual Review of Clinical Psychology, 11, 283-307.

Rae, C., Lee, M.A., Dixon, R.M., Blamire, A.M., Thompson, C.H., Styles, P., ... Stein, J.F. (1998). Metabolic abnormalities in developmental dyslexia detected by $1 \mathrm{H}$ magnetic resonance spectroscopy. The Lancet, 351(9119), 1849-1852.

Ramus, F., \& Szenkovits, G. (2008). What phonological deficit? Quarterly Journal of Experimental Psychology, 61(1), 129-141.

Raven, J., Court, J.H., \& Raven, J. (1992). Standard Progressive Matrices. Oxford, England: Oxford Psychologists Press.

Rothermich, K., \& Kotz, S.A. (2013). Predictions in speech comprehension: fMRI evidence on the meter-semantic interface. NeuroImage, 70, 89-100.

Samuel, A.G., \& Kraljic, T. (2009). Perceptual learning for speech. Attention, Perception, \& Psychophysics, 71(6), 1207-1218.

Schneider, W., Eschman, A., \& Zuccolotto, A. (2002). E-Prime: User's Guide. Pittsburgh, PA: Psychology Software Tools.

Schwab, E.C., Nusbaum, H.C., \& Pisoni, D.B. (1985). Some effects of training on the perception of synthetic speech. Human Factors, 27(4), 395-408.

Shannon, R.V., Zeng, F.-G., Kamath, V., Wygonski, J., \& Ekelid, M. (1995). Speech recognition with primarily temporal cues. Science, 270(5234), 303-304.

Singh, S., Walk, A.M., \& Conway, C.M. (2018). Atypical predictive processing during visual statistical learning in children with developmental dyslexia: an event-related potential study. Annals of Dyslexia, 68(2), 165-179.

Snowling, M. (1998). Dyslexia as a phonological deficit: evidence and implications. Child Psychology and Psychiatry Review, 3(1), 4-11.

Stanberry, L.I., Richards, T.L., Berninger, V.W., Nandy, R.R., Aylward, E.H., Maravilla, K. R., ... Cordes, D. (2006). Lowfrequency signal changes reflect differences in functional connectivity between good readers and dyslexics during continuous phoneme mapping. Magnetic Resonance Imaging, 24(3), 217-229.

Stoodley, C., Ray, N., Jack, A., \& Stein, J. (2008). Implicit learning in control, dyslexic, and garden-variety poor readers. Annals of the New York Academy of Sciences, 1145(1), 173.

Stoodley, C.J. (2014). Distinct regions of the cerebellum show gray matter decreases in autism, ADHD, and developmental dyslexia. Frontiers in Systems Neuroscience, 8, 92.

Stoodley, C.J., Harrison, E.P., \& Stein, J.F. (2006). Implicit motor learning deficits in dyslexic adults. Neuropsychologia, 44(5), 795-798.

Thompson, R., Tanimoto, S., Lyman, R.D., Geselowitz, K., Begay, K.K., Nielsen, K., . . B Berninger, V. (2018). Effective instruction for persisting dyslexia in upper grades: adding hope stories and computer coding to explicit literacy instruction. Education and Information Technologies, 23(3), 1043-1068.

Torgesen, J.K., Rashotte, C.A., \& Wagner, R.K. (1999). TOWRE: Test of Word Reading Efficiency. Ontario: Psychological Corporation Toronto.

Ullman, M.T. (2004). Contributions of memory circuits to language: the declarative/procedural model. Cognition, 92(1-2), 231-270.

Ullman, M.T., Earle, F.S., Walenski, M., \& Janacsek, K. (2020). The neurocognition of developmental disorders of language. Annual Review of Psychology, 71, 389-417.

Van Ingelghem, M., Van Wieringen, A., Wouters, J., Vandenbussche, E., Onghena, P., \& Ghesquière, P. (2001). Psychophysical evidence for a general temporal processing deficit in children with dyslexia. Neuroreport, 12(16), 3603-3607.

van Viersen, S., de Bree, E.H., Verdam, M., Krikhaar, E., Maassen, B., van der Leij, A., \& de Jong, P. F. (2017). Delayed early vocabulary development in children at family risk of dyslexia. Journal of Speech, Language, and Hearing Research, 60(4), 937-949.

Vicari, S., Finzi, A., Menghini, D., Marotta, L., Baldi, S., \& Petrosini, L. (2005). Do children with developmental dyslexia have an implicit learning deficit? Journal of Neurology, Neurosurgery \& Psychiatry, 76(10), 1392-1397.

Vicari, S., Marotta, L., Menghini, D., Molinari, M., \& Petrosini, L. (2003). Implicit learning deficit in children with developmental dyslexia. Neuropsychologia, 41(1), 108-114.

Vroomen, J., Baart, M., Murray, M., \& Wallace, M. (2012). Phonetic recalibration in audiovisual speech. In The Neural Bases of Multisensory Processes, (pp. 363-379). Boca Raton, FL: CRC Press.

Vroomen, J., van Linden, S., De Gelder, B., \& Bertelson, P. (2007). Visual recalibration and selective adaptation in auditory-visual speech perception: contrasting build-up courses. Neuropsychologia, 45(3), 572-577.

Wechsler, D. (1997). Weschsler adult intelligence scale-III. San Antonio, TX: The Psychological Corporation.

Wilson, A.M., \& Lesaux, N.K. (2001). Persistence of phonological processing deficits in college students with dyslexia who have age-appropriate reading skills. Journal of Learning Disabilities, 34(5), 394-400.

Wolf, M., \& Denckla, M.B. (2005). RAN/RAS: Rapid Automatized Naming and Rapid Alternating Stimulus tests. Austin, TX: Pro-ed.

Wolpert, D.M., Diedrichsen, J., \& Flanagan, J.R. (2011). Principles of sensorimotor learning. Nature Reviews Neuroscience, 12(12), 739.

Wolpert, D.M., \& Kawato, M. (1998). Multiple paired forward and inverse models for motor control. Neural Networks, 11(7-8), 1317-1329.

Wolpert, D.M., Miall, R.C., \& Kawato, M. (1998). Internal models in the cerebellum. Trends in Cognitive Sciences, 2(9), 338-347.

Woodcock, R.W. (1987). Woodcock Reading Mastery TestsRevised. Circle Pines, MN: American Guidance Service.

Yang, Y., \& Hong-Yan, B. (2011). Unilateral implicit motor learning deficit in developmental dyslexia. International Journal of Psychology, 46(1), 1-8.

Zeng, F.-G. (2004). Trends in cochlear implants. Trends in Amplification, 8(1), 1-34.

Zhang, X., \& Holt, L.L. (2018). Simultaneous tracking of coevolving distributional regularities in speech. Journal of Experimental Psychology: Human Perception and Performance, 44(11), 1760. 